\title{
BICEP2 and Keck Array: upgrades and improved beam characterization
}

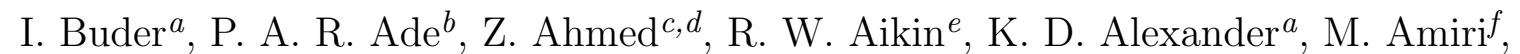
D. Barkats ${ }^{g}$, S. J. Benton ${ }^{h}$, C. A. Bischoff ${ }^{a}$, J. J. Bock ${ }^{e, i}$, J. A. Bonetti ${ }^{i}$, J. A. Brevik ${ }^{e}$, E. Bullock ${ }^{j}$, B. Burger ${ }^{f}$, B. P. Crille,,$i$, G. Davis ${ }^{f}$, C. D. Dowell ${ }^{i}$, L. Duband ${ }^{k}$, J. P. Filippini ${ }^{e}$, S. Fliescher ${ }^{l}$, S. R. Golwala ${ }^{e}$, M. S. Gordon ${ }^{a}$, M. Halpern ${ }^{f}$, M. Hasselfield ${ }^{f}$, S. R. Hildebrandt ${ }^{e, i}$ , G. C. Hilton ${ }^{m}$, V. V. Hristov ${ }^{e}$, H. Hui ${ }^{e}$, K. D. Irwin ${ }^{c, d, m}$, K. S. Karkare ${ }^{a}$, J. P. Kaufman ${ }^{n}$, B. G. Keating ${ }^{n}$, S. Kefelie, S. A. Kernasovskiy ${ }^{c}$, J. M. Kovac ${ }^{a}$, C. L. Kuo ${ }^{c, d}$, E. M. Leitch ${ }^{o}$, M. Lueker ${ }^{e}$, P. Mason ${ }^{e}$, K. G. Megerian ${ }^{i}$, C. B. Netterfield ${ }^{h, p}$, H. T. Nguyen ${ }^{i}$, R. O'Brient ${ }^{i}$, R. W. Ogburn IV ${ }^{c, d}$, A. Orlando ${ }^{n}$, C. Pryke ${ }^{l, j}$, C. D. Reintsema ${ }^{m}$, S. Richter ${ }^{a}$, R. Schwarz ${ }^{l}$, C. D. Sheehy ${ }^{l, o}$, Z. K. Staniszewski ${ }^{e}, i$, R. V. Sudiwala ${ }^{b}$, G. P. Teply ${ }^{e}$, K. L. Thompson ${ }^{c, d}$, J. E. Tolan ${ }^{c}$, A. D. Turner ${ }^{i}$, A. G. Vieregg ${ }^{a, o}$, A. C. Weber ${ }^{i, e}$, D. V. Wiebe ${ }^{f}$, P. Wilson ${ }^{i}$, C. L. Wong ${ }^{a}$, and K. W. Yoon ${ }^{c, d}$

${ }^{a}$ Harvard-Smithsonian Center for Astrophysics, 60 Garden St. MS 42, Cambridge, Massachusetts 02138, USA

${ }^{b}$ School of Physics and Astronomy, Cardiff University, Cardiff, CF24 3AA, United Kingdom

${ }^{c}$ Department of Physics, Stanford University, Stanford, California 94305, USA

${ }^{d}$ Kavli Institute for Particle Astrophysics and Cosmology, SLAC National Accelerator

Laboratory, 2575 Sand Hill Rd, Menlo Park, California 94025, USA

${ }^{e}$ Department of Physics, California Institute of Technology, Pasadena, California 91125, USA

${ }_{f}$ Department of Physics and Astronomy, University of British Columbia, Vancouver, British Columbia, V6T 1Z1, Canada

${ }^{g}$ Joint ALMA Observatory, Vitacura, Santiago, Chile

${ }^{h}$ Department of Physics, University of Toronto, Toronto, Ontario, M5S 1A7, Canada

${ }^{i}$ Jet Propulsion Laboratory, Pasadena, California 91109, USA

${ }^{j}$ Minnesota Institute for Astrophysics, University of Minnesota, Minneapolis, Minnesota 55455, USA

${ }^{k}$ Service des Basses Températures, Commissariat à l'Energie Atomique, 38054 Grenoble, France

${ }^{l}$ Department of Physics, University of Minnesota, Minneapolis, Minnesota 55455, USA

${ }^{m}$ National Institute of Standards and Technology, Boulder, Colorado 80305, USA

${ }^{n}$ Department of Physics, University of California at San Diego, La Jolla, California 92093, USA

${ }^{\circ}$ University of Chicago, Chicago, Illinois 60637, USA

${ }^{p}$ Canadian Institute for Advanced Research, Toronto, Ontario, M5G 1Z8, Canada

\begin{abstract}
Searching for evidence of inflation by measuring B-modes in the cosmic microwave background (CMB) polarization at degree angular scales remains one of the most compelling experimental challenges in cosmology. BICEP2 and the Keck Array are part of a program of experiments at the South Pole whose main goal is to achieve the sensitivity and systematic control necessary for measurements of the tensor-to-scalar ratio at $\sigma(r) \lesssim 0.01$. Beam imperfections that are not sufficiently accounted for are a potential source of spurious polarization that could interfere with that goal. The strategy of BICEP2 and the Keck Array is to completely characterize their telescopes' polarized beam response with a combination of in-lab, pre-deployment, and on-site calibrations. We
\end{abstract}

Send correspondence to I. Buder, ibuder@cfa.harvard.edu

Millimeter, Submillimeter, and Far-Infrared Detectors and Instrumentation for Astronomy VII, edited by Wayne S. Holland, Jonas Zmuidzinas, Proc. of SPIE Vol. 9153, 915312 · @ 2014

SPIE · CCC code: $0277-786 \mathrm{X} / 14 / \$ 18 \cdot$ doi: $10.1117 / 12.2055713$ 
report the status of these experiments, focusing on continued improved understanding of their beams. Far-field measurements of the BICEP2 beam with a chopped thermal source, combined with analysis improvements, show that the level of residual beam-induced systematic errors is acceptable for the goal of $\sigma(r) \sim 0.01$ measurements. Beam measurements of the Keck Array side lobes helped identify a way to reduce optical loading with interior cold baffles, which we installed in late 2013. These baffles reduced total optical loading, leading to a $\sim 10 \%$ increase in mapping speed for the 2014 observing season. The sensitivity of the Keck Array continues to improve: for the 2013 season it was $9.5 \mu \mathrm{K} \sqrt{\mathrm{s}}$ noise equivalent temperature (NET). In 2014 we converted two of the $150-\mathrm{GHz}$ cameras to $100 \mathrm{GHz}$ for foreground separation capability. We have shown that the BICEP2 and the Keck Array telescope technology is sufficient for the goal of $\sigma(r) \sim 0.01$ measurements. Furthermore, the program is continuing with BICEP3, a 100-GHz telescope with 2560 detectors.

Keywords: Inflation, Gravitational waves, Cosmic microwave background, polarization, BICEP2, Keck Array.

\section{INTRODUCTION}

Measurement of cosmic microwave background (CMB) polarization is one of the most promising probes of the inflationary epoch of the early Universe. BICEP2 and the Keck Array are part of a series of experiments whose goal is to measure the degree-angular-scale B-mode (odd-parity) polarization signal predicted by inflation. Cosmologists parameterize the amplitude of the inflationary signal by the tensor-to-scalar ratio, $r$. In the detection of degree-scale B-mode polarization by BICEP2, ${ }^{1}$ we considered the potential systematic errors on $r$ due to telescope beam imperfections and showed them to be negligible. To do so, we used a strategy of completely characterizing the polarized beam of each experiment followed by reducing the spurious polarization effects in analysis. The Keck Array is still observing, and we continue to make improvements to it. These proceedings discuss upgrades made for the 2013 and 2014 seasons of the Keck Array and the status of beam characterization for both experiments.

The inflationary paradigm, by positing an exponential expansion of the early Universe $\left(\lesssim 10^{-36} \mathrm{~s}\right)$, sets the initial conditions for the hot big bang. Inflation is compelling because it naturally solves the flatness, horizon, smoothness, entropy, and monopole problems of standard cosmology. ${ }^{2}$ Furthermore, inflation explains the initial perturbations of the Universe as quantum fluctuations that were stretched by the exponential expansion. A unique prediction of inflation is the production of a stochastic background of gravitational waves. The presence of these gravitational waves at the CMB last scattering surface results in a curl-type (B-mode) polarization pattern at degree angular scales. ${ }^{3}$ (Gravitational waves also generate E-mode polarization, but the inflationary E-mode signature is much smaller than the E-mode polarization from density perturbations. Density perturbations do not produce B-mode polarization at first order.) The amplitude of this pattern is proportional to $r$, which is also proportional to the energy scale at which inflation occured. Measuring this signal is the main science goal of a series of experiments at Amundsen-Scott South Pole Station, including Bicep2 and the Keck Array.

BICEP2 and the Keck Array share many aspects of their design. Both use small (26-cm) aperture cryogenic refracting telescopes. Absorbing, ambient-temperature forebaffles block potential pickup of the ground or Galaxy. The detectors are planar arrays of antenna-coupled transition-edge sensor (TES) bolometers. BICEP2 has 512 such bolometers in its focal plane, and they are paired into 256 pixels where each pixel has two bolometers with orthogonally polarized antennas. The Keck Array has five BICEP2-style receivers in a close-packed configuration. Both experiments have three-axis mount systems; we refer to rotation around the telescope boresight as "deck" rotation. More details about the instruments are in previous publications. ${ }^{4-8}$

The inflationary signal is very small compared to potential contaminating signals such as the CMB temperature and E-mode fluctuations, Galaxy, and ground. Beam imperfections, particularly mismatches between the two detectors in the same pixel, can create spurious polarization and contaminate the B-mode measurement. Section 2 covers characterization of the BICEP2 and the Keck Array beams, including the measurements leading to the limit on BICEP2 beam-induced systematic errors at $r<0.003$. We continue to improve the sensitivity and instrumental control of systematics of the Keck Array. Section 3 covers improvements in sensitivity, differential pointing, forebaffle loading, and the addition of 100-GHz receivers. 


\section{IMPROVED BEAM CHARACTERIZATION OF BICEP2 AND KECK ARRAY}

We have previously reported beam characterization of BICEP $2^{9}$ and the Keck Array. ${ }^{10}$ Since then we have made beam measurements with a brighter microwave source, resulting in a higher signal-to-noise ratio, and improved the corresponding analysis techniques. Finally, we have improved our understanding of the far side lobes of the beams.

\subsection{Main beam characterization}

We measured the BICEP2 optical response in the far field in situ with artificial microwave sources. Using this setup we made multiple maps of each detector's beam. We analyzed and modeled the beams, including the mismatch between orthogonal detectors in the same pixel. The resulting maps and models informed simulations of the systematic effects of beam imperfections on the measurement of $r$.

The far-field beam mapping setup consisted of a microwave source and flat mirror to redirect the radiation into the telescope (Figure 1). For the measurements described in this section, the source was a thermal chopper: Rotating blades, covered in Eccosorb* microwave absorber, alternately presented ambient-temperature $(\sim 250 \mathrm{~K})$ or sky $(\sim 15 \mathrm{~K})$ radiation to the telescope (Figure 2$)$. A flat mirror behind the chopper blades redirected sky radiation from zenith. The typical chopper signal modulation was at $10 \sim 14 \mathrm{~Hz}$ for BICEP2 measurements. Compared to previous measurements, the active source aperture was larger (45-cm diameter). (We used a broadband amplified noise source for some measurements, see Section 2.2.) We mounted the source on top of a 10-m tall mast located on a nearby building, $195 \mathrm{~m}$ away from the BICEP2 telescope. A flat mirror mounted directly above the telescope allowed it to observe the source. (BICEP2 and the Keck Array cannot observe at low enough elevation to see the mast-mounted sources directly.)

Using this setup we made maps of each detector's beam by scanning the telescope over the source in azimuth and elevation. We repeated the mapping procedure at multiple deck angles to confirm consistency and repeatability of the results. We then combined the data from all deck angles (Figure 3). The combined maps show the expected main beam shape, Airy rings, and known cross-talk features (primarily due to cross talk in the readout system).

We constructed an elliptical Gaussian beam model based on the map of each detector. The model parameters were: the location of the beam center, $\vec{r}$; the overall amplitude ("gain"), $g$; the beam width, $\sigma$; and the ellipticity in "plus" and "cross" orientations, $p$ and $c$. The average beam width and scatter among detectors for BICEP2 were $\sigma=0.221^{\circ} \pm 0.006^{\circ}$. We calculated the differential beam parameters for the two detectors in each pixel because they directly correspond to potential spurious polarization sources. Each of these differential parameters corresponds to a mismatch of the orthogonally polarized beams whose difference we used to measure the polarization of the CMB. For one of these effects, differential ellipticity, we used the measured parameters to subtract the effect in analysis. For differential gain and differential pointing, we "deprojected" the effects, an operation that removes them without precise knowledge of their amplitude. ${ }^{11-13}$ We used the beam maps (not the elliptical Gaussian model) as inputs to simulations to calculate the residual spurious polarization after the subtraction and deprojection operations. The residual contamination was equivalent to $r<0.003$.

We used a similar proceedure to measure the Keck Array far-field beams. We used the same thermal source but mounted it on a different mast (located on a different building) so that the source-telescope distance was $211 \mathrm{~m}$. As for Bicep2, we fit an elliptical Gaussian model to the resulting maps. The average Keck Array beam width and scatter among detectors were $\sigma=0.215^{\circ} \pm 0.007^{\circ}$ for 2012 observations. Differential pointing was smaller on average by a factor of $\sim 5$ in the Keck Array than in BICEP2 (although there was significant variation among the different receivers and seasons of the Keck Array). Improvements in the detector design and fabrication process were responsible for this improvement. ${ }^{14}$ Simulations of the potential impact of beam mismatch in the Keck Array are in progress.

\footnotetext{
*http://www.eccosorb.com/
} 


\subsection{Far side lobe characterization}

We paid special attention to far side lobes of the beam, which we considered to be the part of the beam pattern that could potentially pick up the Galaxy or ground ( $\gtrsim 15^{\circ}$ from the main beam). We used a two-stage mitigation strategy consisting of an absorbing, comoving forebaffle and a fixed, reflecting ground shield to limit the effects of the far side lobes intrinsic to the telescope. We measured the effectiveness of the forebaffle in two ways: by measuring the change in optical loading upon installing it and by measuring the side lobes with an amplified noise source.

We measured the total power in far side lobes by comparing the detector optical loading with and without the forebaffle installed. When the forebaffle was installed, optical rays that would have otherwise terminated on the cold sky instead terminated on the warm forebaffle; this increased total loading by $3 \sim 6 \mathrm{~K}_{\mathrm{CMB}}$ for BICEP2. The forebaffle loading was higher for the Keck Array $\left(5 \sim 10 \mathrm{~K}_{\mathrm{CMB}}\right)$. Both had a pattern of higher loading for pixels near the center of the focal plane. Furthermore, this loading was higher than in BICEP1 ${ }^{15}\left(<2 \mathrm{~K}_{\mathrm{CMB}}\right)$. We found the major source of additional forebaffle loading in the Keck Array and were able to improve it for 2014 observations by adding interior cold baffles (Section 3.2).

To measure the spatial pattern of the far side lobes we used a modified beam mapping procedure. We created an amplified noise source from the Johnson noise of a $50-\Omega$ resistor. A series of amplifiers, frequency multipliers, and filters brought the output to a broadband frequency range of $140 \sim 160 \mathrm{GHz}$. For the Keck Array we used an additional noise source with a band near $100 \mathrm{GHz}$ (Figure 4). The source was linearly polarized, allowing measurement of side-lobe polarization. We mounted the sources on a mast near BICEP2 (10 m away) or the Keck Array (20 m away). These were the same masts as used for the main beam mapping; however, for the far-side-lobe mapping we used the mast located on the same building as each telescope to map a larger area without using a mirror. In this configuration, we aimed the source down at the telescope at $45 \sim 60^{\circ}$ from horizontal. We scanned the telescope to achieve nearly full coverage up to $90^{\circ}$ from the main beam. We repeated such observations with various source polarizations and attenuations and with the forebaffles on and off. Combining data from different source attenuations we made maps with $\sim 70 \mathrm{~dB}$ dynamic range. In BICEP2 we found no sharp features in the far side lobes; however, we detected some diffuse power far from the main beam. With the forebaffle on, the region $>25^{\circ}$ from the main beam contained $\lesssim 0.1 \%$ of the total integrated power. By comparing maps made with and without the forebaffle, we calculated that the average fraction of power intercepted by the forebaffle was $0.7 \%$. This corresponds to $3 \mathrm{~K}_{\mathrm{CMB}}$, consistent with the increase in detector loading discussed above.
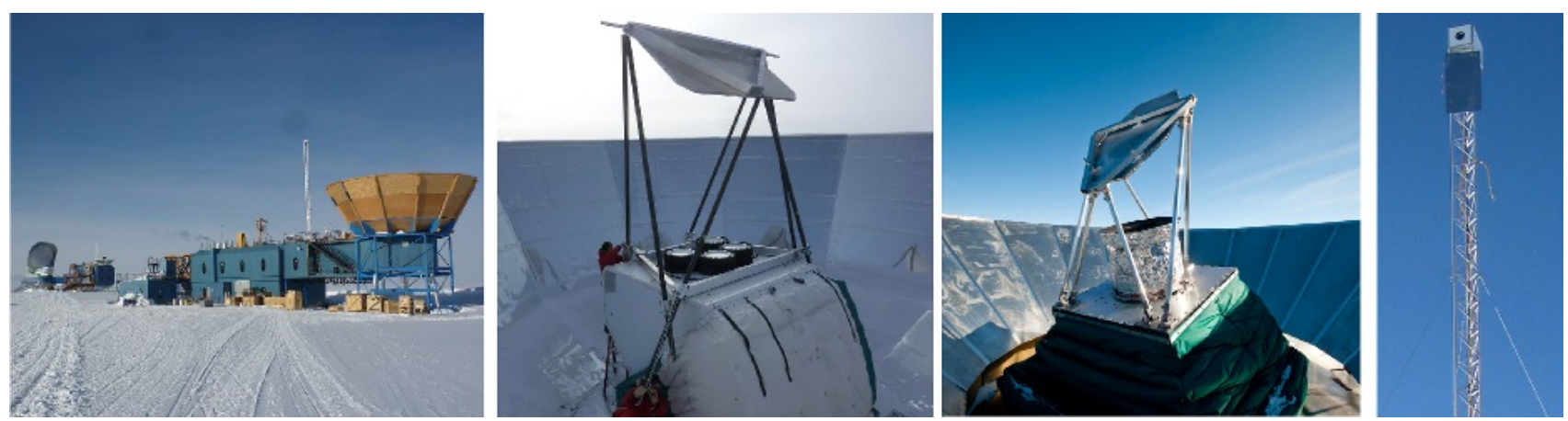

Figure 1. To make far-field beam maps we put microwave sources on masts and used flat mirrors to redirect the radiation into our telescopes. Left: The Dark Sector Laboratory (background) and Martin A. Pomerantz Observatory (foreground), housing BICEP2 and the Keck Array, respectively. Each building had a mast for far-field beam mapping, and the distance from a source on one building to the telescope on the other building was $\approx 200 \mathrm{~m}$. Center Left: A flat mirror installed above the Keck Array. Center Right: A different flat mirror installed above BICEP2. Right: We installed various microwave sources on top of the masts for different beam mapping goals; see Section 2 for details.

\section{KECK ARRAY UPGRADES}

We have the opportunity to upgrade the configuration of the Keck Array every year. ${ }^{6,7,10,14,16,17}$ For the 2013 observing season, we replaced some detectors to improve their sensitivity. For the 2014 observing season, we made 


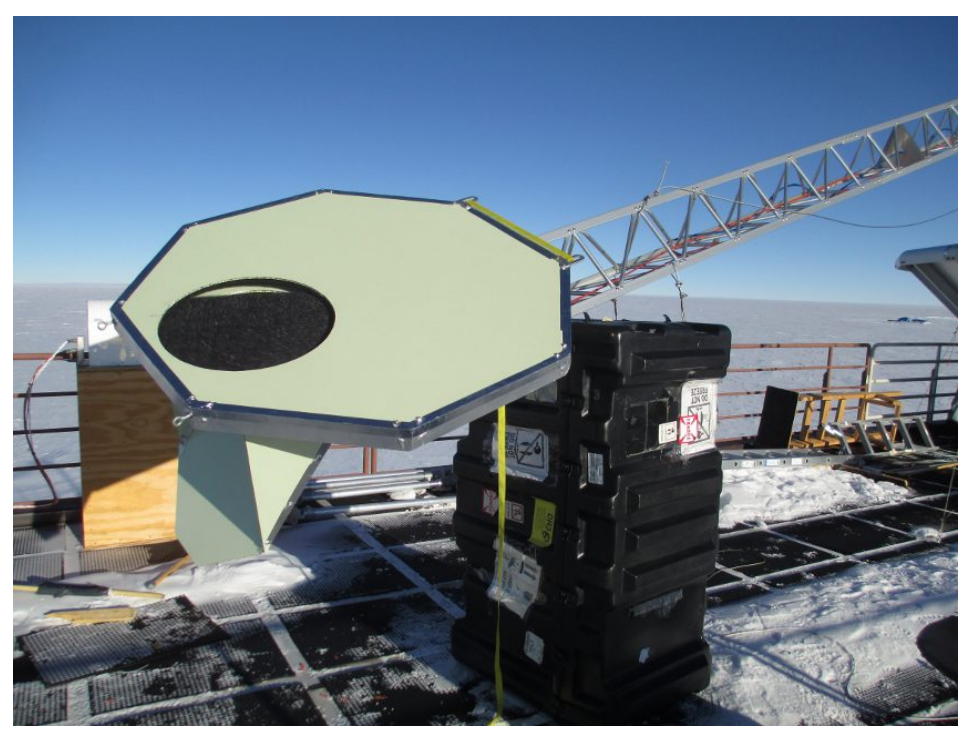

Figure 2. We characterized the main beams of BICEP2 and the Keck Array using a chopped thermal source. Rotating blades alternately presented sky $(\sim 15 \mathrm{~K})$ and ambient-temperature $(\sim 250 \mathrm{~K})$ radiation to the telescope. A flat mirror behind the chopper blades redirected sky radiation from zenith. The large active source aperture (45-cm diameter) provided high signal-to-noise.

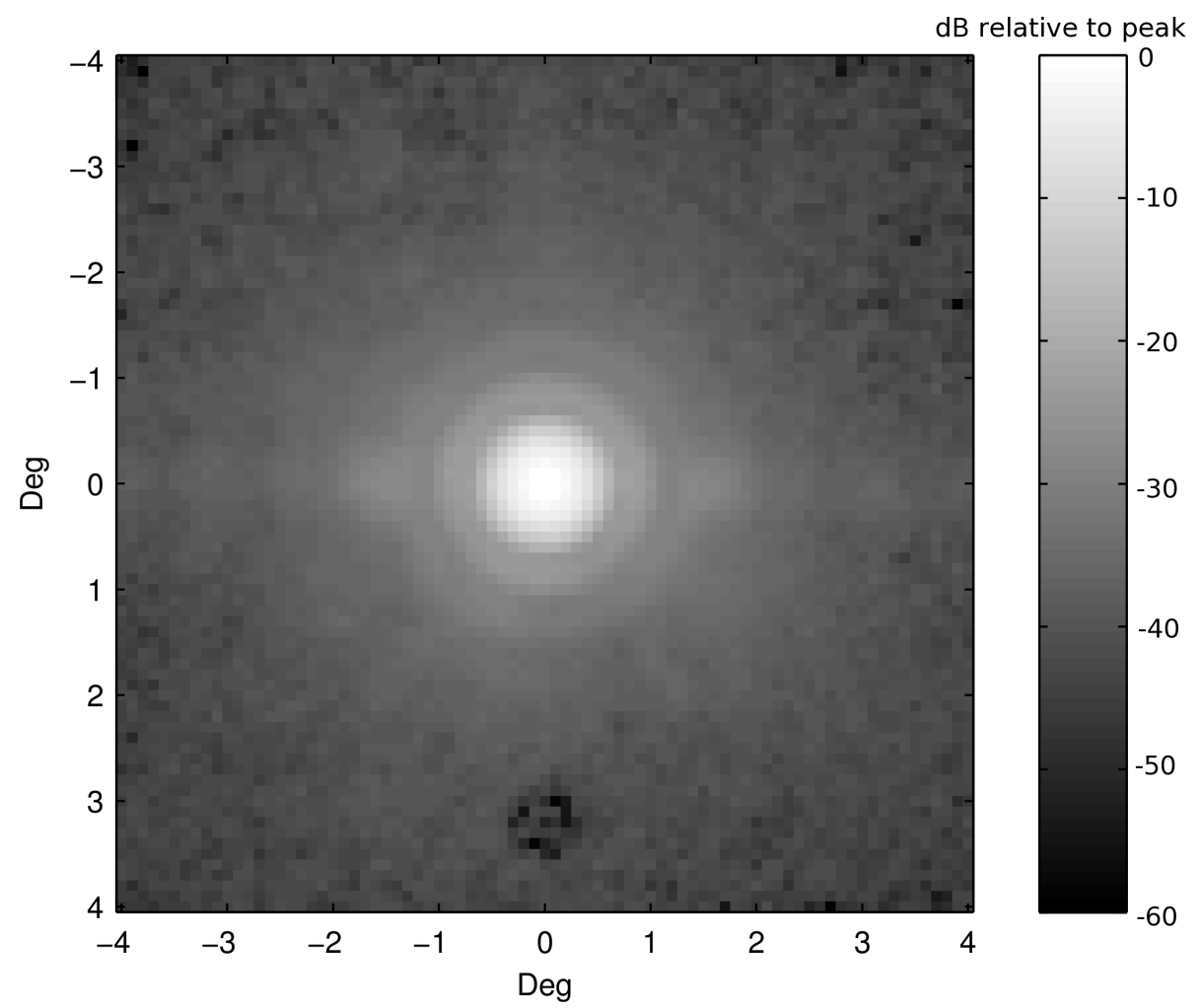

Figure 3. We combined the beam maps from all functional BICEP2 detectors to make an average beam map. The main beam shape and Airy ring structure were consistent with simulations of the optics. The additional beam features at $1.5^{\circ}$ and $3^{\circ}$ from the peak were primarily due to cross talk in the readout system. 


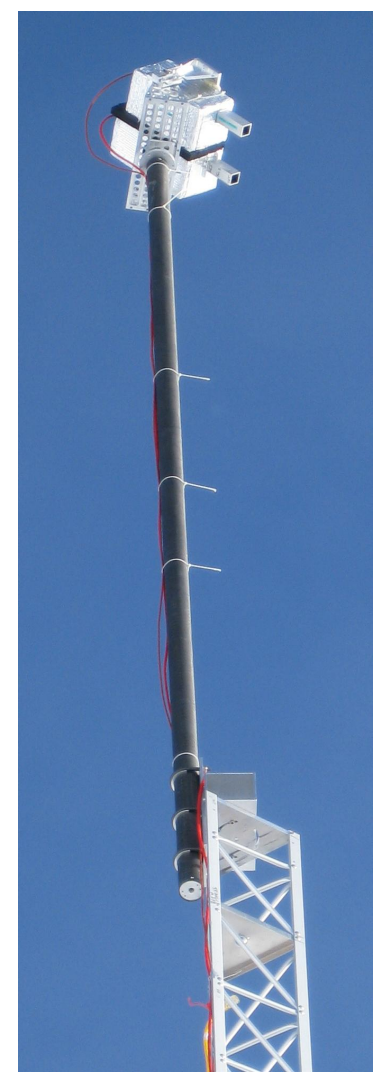

Figure 4. We used amplified noise sources to measure the spatial pattern of the far side lobes. Shown here is the source setup for the Keck Array. To simultaneously map the $150-\mathrm{GHz}$ and $100-\mathrm{GHz}$ receivers, we mounted two sources on the same mast, one tuned for each frequency band.

two main upgrades: first, we reduced the forebaffle loading based on the results of the measurements described in Section 2.2; second, we replaced two 150-GHz receivers with new 100-GHz receivers. Multi-frequency coverage is essential for distinguishing the CMB from Galactic foregrounds, ${ }^{18}$ especially in light of the B-mode detection by BICEP2.

\subsection{Sensitivity improvements}

In preparation for the 2013 observing season, we replaced detector tiles ${ }^{\dagger}$ measured to have sub-optimal sensitivity. We replaced all tiles from the two receivers with the worst sensitivity in the 2012 season. The replacements were the BICEP2 focal plane, known to have a noise equivalent temperature (NET, in CMB temperature units) of $15.8 \mu \mathrm{K} \sqrt{\mathrm{s}}$, and a newly fabricated focal plane, measured to have high optical efficiency in the lab $(40 \sim 50 \%)$. Finally, we replaced one tile (i.e. $25 \%$ of the detectors) in a third receiver because that tile had unusual, nonGaussian noise properties in 2012 data. The combined NET for all Keck Array receivers in 2012 was $11.5 \mu \mathrm{K} \sqrt{\mathrm{s}}$, calculated using the same method as in BICEP2 ${ }^{17}$ Because of the detector replacements, the 2013 NET improved to $9.5 \mu \mathrm{K} \sqrt{\mathrm{s}}$.

\subsection{Reduction of forebaffle loading}

We found that forebaffle thermal emission was contributing an unnecessarily high $5 \sim 10 \mathrm{~K}_{\mathrm{CMB}}$ to the detector loading (Section 2.2). Based on on-site and in-lab measurements, we identified the cause as shallow-incidence reflections off the inner (4-K) wall of the telescope. We blackened the telescope walls of both BICEP2 and the Keck Array with carbon-loaded Stycast 2850 FT epoxy applied to Eccosorb HR-10 microwave absorber.

\footnotetext{
†Each receiver's focal plane has four detector tiles.
} 
However, we roughened the surface texture of the HR-10 for BICEP2, but did not do so for the Keck Array. Based on lab measurements, the reflectance at shallow incidence angles $\left(15-20^{\circ}\right)$ was up to $\sim 5$ times higher for the non-roughened surface used in the Keck Array. We concluded that the additional forebaffle loading in the Keck Array was due to emission from the forebaffle reflecting off the telescope walls and onto the detectors.

To reduce this forebaffle loading, we upgraded the Keck Array telescopes with baffles, blackened rings placed to intercept shallow-incidence reflections. The baffles were thin aluminum annuli oriented perpendicular to the telescope walls so that any rays at shallow incidence to the walls would be at near-normal incidence to the baffles (Figure 5). We covered the baffles with the same (non-roughened) absorber and epoxy mixture used on the walls. Each telescope had six baffles, evenly spaced between the two lenses. We set the baffle inner diameters so they would not intercept the detector main beams. The baffles were heat sunk to the 4-K telescope walls, so the increase in loading due to emission from the baffles was negligible. We installed these baffles on all Keck Array receivers in preparation for the 2014 season. With the baffles, the forebaffle loading was $2 \sim 4 \mathrm{~K}_{\mathrm{CMB}}$. This loading reduction corresponded to a $5 \sim 10 \%$ improvement in NET due to the installation of the baffles.

\subsection{Addition of 100-GHz receivers}

For the 2014 season, we changed the observing band of two receivers from $150 \mathrm{GHz}$ to $100 \mathrm{GHz}$. This change required replacement of the focal planes, lenses, and optical filters. The 100-GHz focal planes each had 144 dual-polarization pixels (288 bolometers). The decrease compared to $150 \mathrm{GHz}$ was due to scaling the design to the larger wavelength; a smaller number of pixels fit in the same focal plane area. We used the same lens and filter design as $150 \mathrm{GHz}$. We changed the anti-reflection coating layer thicknesses to optimize for the new frequency band, and we used lower-cutoff $\left(4-\mathrm{cm}^{-1}, 120-\mathrm{GHz}\right)$ metal mesh low-pass filters ${ }^{19}$ to eliminate response to submillimeter radiation. Based on on-site Fourier transform spectroscopy (FTS) measurements, the average center frequency and bandwidth were $94.8 \pm 0.8 \mathrm{GHz}$ and $25.5 \pm 0.4 \mathrm{GHz}$, respectively. ${ }^{20}$ (Errors are the scatter among detectors.)

The Keck Array 2014 CMB science observations began in March, and the 100-GHz receivers have been performing well. Even with only $\sim 3$ months of data, the map depth from the Keck Array at $100 \mathrm{GHz}$ was already better than from three years of BICEP1 100-GHz data. Analysis of these data is in progress.
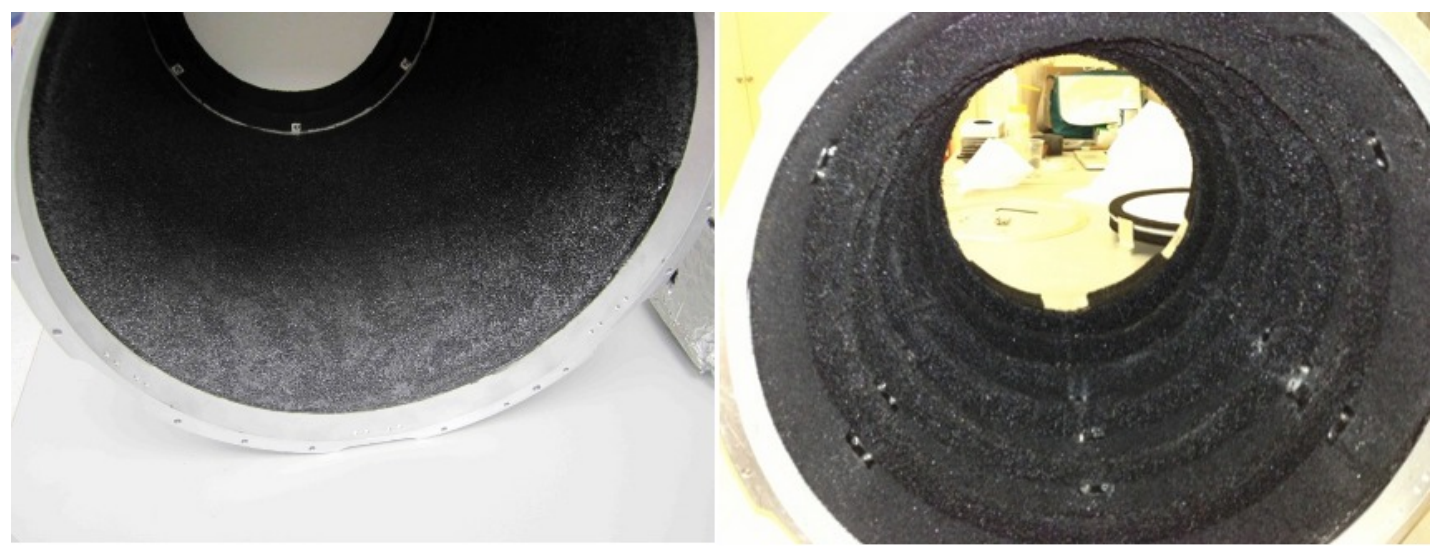

Figure 5. In order to reduce detector loading from the warm forebaffles, we added baffles inside the cold telescope tubes of the Keck Array for the 2014 season. Left: A Keck Array telescope tube as configured for the 2011-2013 observing seasons. In this configuration, shallow-incidence reflections off the telescope walls allowed a significant amount of forebaffle emission to reach the detectors. Right: A Keck Array telescope tube with baffles added as configured for the 2014 season. We oriented the baffles so that any such shallow-incidence rays would be at near-normal incidence on the baffles and therefore absorbed efficiently on the cold telescope walls.

\section{CONCLUSIONS}

These proceedings have summarized improved beam characterization for BICEP2 and the Keck Array and continued upgrades of the Keck Array. The BICEP2 telescope beams have been measured at high signal-to-noise. 
Those beam measurements were a critical input to the simulations that showed the residual uncertainty in the BICEP2 B-mode detection from beam-related systematic effects was equivalent to $r<0.003$. Measurements of far side lobes revealed an opportunity to reduce the detector optical loading in Keck Array; we upgraded the telescopes with internal cold baffles and confirmed the loading reduction. In 2013, after detector upgrades, the Keck Array sensitivity at $150 \mathrm{GHz}$ was $9.5 \mu \mathrm{K} \sqrt{\mathrm{s}}$. In 2014 we installed two 100-GHz receivers. In late 2014 we will deploy BICEP3, a 100-GHz telescope with 2560 detectors, ${ }^{21}$ dramatically increasing the $100-\mathrm{GHz}$ sensitivity of the BICEP/Keck Array program. The resulting data will greatly improve our ability to distinguish CMB B-modes from foregrounds.

\section{ACKNOWLEDGMENTS}

The Bicep2 and the Keck Array projects have been made possible through support from the National Science Foundation (grant Nos. ANT-0742818, ANT-0742592, ANT-1044978, ANT-1110087, and ANT-1145172), the W. M. Keck Foundation, the Canada Foundation for Innovation, and the British Columbia Development Fund. The development of antenna-coupled detector technology was supported by the JPL Research and Technology Development Fund and grants 06-ARPA206-0040 and 10-SAT10-0017 from the NASA APRA and SAT programs. The development and testing of focal planes were supported by the Gordon and Betty Moore Foundation at Caltech. The computations in these proceedings were run on the Odyssey cluster supported by the FAS Science Division Research Computing Group at Harvard University. Tireless administrative support was provided by Irene Coyle and Kathy Deniston.

We are grateful to Steffen Richter as our 2010-2012 BICEP2 winter-over, and to Robert Schwarz as our 2011-2014 Keck Array winter-over. We thank the staff of the US Antarctic Program and in particular the South Pole Station without whose help this research would not have been possible. We thank our BICEP1 and SPIDER colleagues for useful discussions and shared expertise.

\section{REFERENCES}

[1] BICEP2 Collaboration, Ade, P. A. R., Aikin, R. W., Barkats, D., Benton, S. J., Bischoff, C. A., Bock, J. J., Brevik, J. A., Buder, I., Bullock, E., Dowell, C. D., Duband, L., Filippini, J. P., Fliescher, S., Golwala, S. R., Halpern, M., Hasselfield, M., Hildebrandt, S. R., Hilton, G. C., Hristov, V. V., Irwin, K. D., Karkare, K. S., Kaufman, J. P., Keating, B. G., Kernasovskiy, S. A., Kovac, J. M., Kuo, C. L., Leitch, E. M., Lueker, M., Mason, P., Netterfield, C. B., Nguyen, H. T., O'Brient, R., Ogburn, IV, R. W., Orlando, A., Pryke, C., Reintsema, C. D., Richter, S., Schwarz, R., Sheehy, C. D., Staniszewski, Z. K., Sudiwala, R. V., Teply, G. P., Tolan, J. E., Turner, A. D., Vieregg, A. G., Wong, C. L., and Yoon, K. W., "BICEP2 I: Detection Of B-mode Polarization at Degree Angular Scales," Phys. Rev. Lett. 112, 241101 (2014).

[2] Planck Collaboration, Ade, P. A. R., Aghanim, N., Armitage-Caplan, C., Arnaud, M., Ashdown, M., AtrioBarandela, F., Aumont, J., Baccigalupi, C., Banday, A. J., et al., "Planck 2013 results. XXII. Constraints on inflation," ArXiv e-prints , 1303.5082 (2013).

[3] Polnarev, A. G., "Polarization and Anisotropy Induced in the Microwave Background by Cosmological Gravitational Waves," Soviet Astronomy 29, 607-613 (1985).

[4] BICEP2 Collaboration, Ade, P. A. R., Aikin, R. W., Amiri, M., Barkats, D., Benton, S. J., Bischoff, C. A., Bock, J. J., Brevik, J. A., Buder, I., Bullock, E., Davis, G., Dowell, C. D., Duband, L., Filippini, J. P., Fliescher, S., Golwala, S. R., Halpern, M., Hasselfield, M., Hildebrandt, S. R., Hilton, G. C., Hristov, V. V., Irwin, K. D., Karkare, K. S., Kaufman, J. P., Keating, B. G., Kernasovskiy, S. A., Kovac, J. M., Kuo, C. L., Leitch, E. M., Llombart, N., Lueker, M., Netterfield, C. B., Nguyen, H. T., O'Brient, R., Ogburn, IV, R. W., Orlando, A., Pryke, C., Reintsema, C. D., Richter, S., Schwarz, R., Sheehy, C. D., Staniszewski, Z. K., Story, K. T., Sudiwala, R. V., Teply, G. P., Tolan, J. E., Turner, A. D., Vieregg, A. G., Wilson, P., Wong, C. L., and Yoon, K. W., "BICEP2 II: Experiment and Three-Year Data Set," ArXiv e-prints , $1403.4302(2014)$.

[5] Ogburn, IV, R. W., Ade, P. A. R., Aikin, R. W., Amiri, M., Benton, S. J., Bock, J. J., Bonetti, J. A., Brevik, J. A., Burger, B., Dowell, C. D., Duband, L., Filippini, J. P., Golwala, S. R., Halpern, M., Hasselfield, M., Hilton, G., Hristov, V. V., Irwin, K., Kaufman, J. P., Keating, B. G., Kovac, J. M., Kuo, C. L., Lange, A. E., Leitch, E. M., Netterfield, C. B., Nguyen, H. T., Orlando, A., Pryke, C. L., Reintsema, C., Richter, S., 
Ruhl, J. E., Runyan, M. C., Sheehy, C. D., Staniszewski, Z. K., Stokes, S. A., Sudiwala, R. V., Teply, G. P., Tolan, J. E., Turner, A. D., Wilson, P., and Wong, C. L., "The BICEP2 CMB polarization experiment," Proc. SPIE 7741, 77411G (2010).

[6] Sheehy, C. D., Ade, P. A. R., Aikin, R. W., Amiri, M., Benton, S., Bischoff, C., Bock, J. J., Bonetti, J. A., Brevik, J. A., Burger, B., Dowell, C. D., Duband, L., Filippini, J. P., Golwala, S. R., Halpern, M., Hasselfield, M., Hilton, G., Hristov, V. V., Irwin, K., Kaufman, J. P., Keating, B. G., Kovac, J. M., Kuo, C. L., Lange, A. E., Leitch, E. M., Lueker, M., Netterfield, C. B., Nguyen, H. T., Ogburn, IV, R. W., Orlando, A., Pryke, C. L., Reintsema, C., Richter, S., Ruhl, J. E., Runyan, M. C., Staniszewski, Z., Stokes, S., Sudiwala, R., Teply, G., Thompson, K. L., Tolan, J. E., Turner, A. D., Wilson, P., and Wong, C. L., "The Keck Array: a pulse tube cooled CMB polarimeter," Proc. SPIE 7741, 77411R (2010).

[7] Ogburn, R. W., Ade, P. A. R., Aikin, R. W., Amiri, M., Benton, S. J., Bischoff, C. A., Bock, J. J., Bonetti, J. A., Brevik, J. A., Bullock, E., Burger, B., Davis, G., Dowell, C. D., Duband, L., Filippini, J. P., Fliescher, S., Golwala, S. R., Gordon, M., Halpern, M., Hasselfield, M., Hilton, G., Hristov, V. V., Hui, H., Irwin, K., Kaufman, J. P., Keating, B. G., Kernasovskiy, S. A., Kovac, J. M., Kuo, C. L., Leitch, E. M., Lueker, M., Montroy, T., Netterfield, C. B., Nguyen, H. T., O'Brient, R., Orlando, A., Pryke, C. L., Reintsema, C., Richter, S., Ruhl, J. E., Runyan, M. C., Schwarz, R., Sheehy, C. D., Staniszewski, Z. K., Sudiwala, R. V., Teply, G. P., Thompson, K., Tolan, J. E., Turner, A. D., Vieregg, A. G., Wiebe, D. V., Wilson, P., and Wong, C. L., "BICEP2 and Keck array operational overview and status of observations," Proc. SPIE $\mathbf{8 4 5 2}$, 84521A (2012).

[8] Staniszewski, Z., Aikin, R. W., Amiri, M., Benton, S. J., Bischoff, C., Bock, J. J., Bonetti, J. A., Brevik, J. A., Burger, B., Dowell, C. D., Duband, L., Filippini, J. P., Golwala, S. R., Halpern, M., Hasselfield, M., Hilton, G., Hristov, V. V., Irwin, K., Kovac, J. M., Kuo, C. L., Lueker, M., Montroy, T., Nguyen, H. T., Ogburn, R. W., O’Brient, R., Orlando, A., Pryke, C., Reintsema, C., Ruhl, J. E., Schwarz, R., Sheehy, C., Stokes, S., Thompson, K. L., Teply, G., Tolan, J. E., Turner, A. D., Vieregg, A. G., Wilson, P., Wiebe, D., and Wong, C. L., "The Keck Array: A Multi Camera CMB Polarimeter at the South Pole," Journal of Low Temperature Physics 167, 827-833 (2012).

[9] Aikin, R. W., Ade, P. A., Benton, S., Bock, J. J., Bonetti, J. A., Brevik, J. A., Dowell, C. D., Duband, L., Filippini, J. P., Golwala, S. R., Halpern, M., Hristov, V. V., Irwin, K., Kaufman, J. P., Keating, B. G., Kovac, J. M., Kuo, C. L., Lange, A. E., Netterfield, C. B., Nguyen, H. T., Ogburn, IV, R. W., Orlando, A., Pryke, C., Richter, S., Ruhl, J. E., Runyan, M. C., Sheehy, C., Stokes, S. A., Sudiwala, R., Teply, G. P., Tolan, J. E., Turner, A. D., Wilson, P., and Wong, C. L., "Optical performance of the BICEP2 Telescope at the South Pole," Proc. SPIE 7741, 77410V (2010).

[10] Vieregg, A. G., Ade, P. A. R., Aikin, R., Bischoff, C., Bock, J. J., Bonetti, J. A., Bradford, K. J., Brevik, J. A., Dowell, C. D., Duband, L., Filippini, J. P., Fliescher, S., Golwala, S. R., Gordon, M. S., Halpern, M., Hilton, G., Hristov, V. V., Irwin, K., Kernasovskiy, S., Kovac, J. M., Kuo, C. L., Leitch, E., Lueker, M., Montroy, T., Netterfield, C. B., Nguyen, H. T., O’Brient, R., Ogburn, R. W., Pryke, C., Ruhl, J. E., Runyan, M., Schwarz, R., Sheehy, C., Staniszewski, Z., Sudiwala, R., Teply, G., Tolan, J., Turner, A. D., Wilson, P., and Wong, C. L., "Optical characterization of the Keck array polarimeter at the South Pole," Proc. SPIE 8452, 845226 (2012).

[11] Barkats, D., Aikin, R., Bischoff, C., Buder, I., Kaufman, J. P., Keating, B. G., Kovac, J. M., Su, M., Ade, P. A. R., Battle, J. O., Bierman, E. M., Bock, J. J., Chiang, H. C., Dowell, C. D., Duband, L., Filippini, J., Hivon, E. F., Holzapfel, W. L., Hristov, V. V., Jones, W. C., Kuo, C. L., Leitch, E. M., Mason, P. V., Matsumura, T., Nguyen, H. T., Ponthieu, N., Pryke, C., Richter, S., Rocha, G., Sheehy, C., Kernasovskiy, S. S., Takahashi, Y. D., Tolan, J. E., and Yoon, K. W., "Degree-scale Cosmic Microwave Background Polarization Measurements from Three Years of BICEP1 Data," ApJ 783, 67 (2014).

[12] Aikin, R. W., "Testing inflationary cosmology with the BICEP1 and BICEP2 experiments," (2013). California Institute of Technology Ph.D. Thesis.

[13] Sheehy, C. D., "Progress toward a detection of inflationary B-modes with the BICEP2 and Keck Array polarimeters," (2013). University of Chicago Ph.D. Thesis.

[14] O'Brient, R., Ade, P. A. R., Ahmed, Z., Aikin, R. W., Amiri, M., Benton, S., Bischoff, C., Bock, J. J., Bonetti, J. A., Brevik, J. A., Burger, B., Davis, G., Day, P., Dowell, C. D., Duband, L., Filippini, J. P., Fliescher, S., Golwala, S. R., Grayson, J., Halpern, M., Hasselfield, M., Hilton, G., Hristov, V. V., Hui, 
H., Irwin, K., Kernasovskiy, S., Kovac, J. M., Kuo, C. L., Leitch, E., Lueker, M., Megerian, K., Moncelsi, L., Netterfield, C. B., Nguyen, H. T., Ogburn, R. W., Pryke, C. L., Reintsema, C., Ruhl, J. E., Runyan, M. C., Schwarz, R., Sheehy, C. D., Staniszewski, Z., Sudiwala, R., Teply, G., Tolan, J. E., Turner, A. D., Tucker, R. S., Vieregg, A., Wiebe, D. V., Wilson, P., Wong, C. L., Wu, W. L. K., and Yoon, K. W., "Antenna-coupled TES bolometers for the Keck array, Spider, and Polar-1," Proc. SPIE 8452, 84521G (2012).

[15] Takahashi, Y. D., Ade, P. A. R., Barkats, D., Battle, J. O., Bierman, E. M., Bock, J. J., Chiang, H. C., Dowell, C. D., Duband, L., Hivon, E. F., Holzapfel, W. L., Hristov, V. V., Jones, W. C., Keating, B. G., Kovac, J. M., Kuo, C. L., Lange, A. E., Leitch, E. M., Mason, P. V., Matsumura, T., Nguyen, H. T., Ponthieu, N., Pryke, C., Richter, S., Rocha, G., and Yoon, K. W., "Characterization of the BICEP Telescope for High-precision Cosmic Microwave Background Polarimetry," ApJ 711, 1141-1156 (2010).

[16] Orlando, A., Aikin, R. W., Amiri, M., Bock, J. J., Bonetti, J. A., Brevik, J. A., Burger, B., Chattopadthyay, G., Day, P. K., Filippini, J. P., Golwala, S. R., Halpern, M., Hasselfield, M., Hilton, G. C., Irwin, K. D., Kenyon, M., Kovac, J. M., Kuo, C. L., Lange, A. E., Leduc, H. G., Llombart, N., Nguyen, H. T., Ogburn, R. W., Reintsema, C. D., Runyan, M. C., Staniszewski, Z., Sudiwala, R., Teply, G., Trangsrud, A. R., Turner, A. D., and Wilson, P., "Antenna-coupled TES bolometer arrays for BICEP2/Keck and SPIDER," Proc. SPIE 7741, 77410H (2010).

[17] Kernasovskiy, S., Ade, P. A. R., Aikin, R. W., Amiri, M., Benton, S., Bischoff, C., Bock, J. J., Bonetti, J. A., Brevik, J. A., Burger, B., Davis, G., Dowell, C. D., Duband, L., Filippini, J. P., Fliescher, S., Golwala, S. R., Halpern, M., Hasselfield, M., Hilton, G., Hristov, V. V., Irwin, K., Kovac, J. M., Kuo, C. L., Leitch, E., Lueker, M., Netterfield, C. B., Nguyen, H. T., O’Brient, R., Ogburn, R. W., Pryke, C. L., Reintsema, C., Ruhl, J. E., Runyan, M. C., Schwarz, R., Sheehy, C. D., Staniszewski, Z., Sudiwala, R., Teply, G., Tolan, J. E., Turner, A. D., Vieregg, A., Wiebe, D. V., Wilson, P., and Wong, C. L., "Optimization and sensitivity of the Keck array," Proc. SPIE 8452, 84521B (2012).

[18] Dunkley, J., Amblard, A., Baccigalupi, C., Betoule, M., Chuss, D., Cooray, A., Delabrouille, J., Dickinson, C., Dobler, G., Dotson, J., Eriksen, H. K., Finkbeiner, D., Fixsen, D., Fosalba, P., Fraisse, A., Hirata, C., Kogut, A., Kristiansen, J., Lawrence, C., Magalhaẽs, A. M., Miville-Deschenes, M. A., Meyer, S., Miller, A., Naess, S. K., Page, L., Peiris, H. V., Phillips, N., Pierpaoli, E., Rocha, G., Vaillancourt, J. E., and Verde, L., "Prospects for polarized foreground removal," in [American Institute of Physics Conference Series], Dodelson, S., Baumann, D., Cooray, A., Dunkley, J., Fraisse, A., Jackson, M. G., Kogut, A., Krauss, L., Zaldarriaga, M., and Smith, K., eds., American Institute of Physics Conference Series 1141, 222-264 (2009).

[19] Ade, P. A. R., Pisano, G., Tucker, C., and Weaver, S., "A review of metal mesh filters," Proc. SPIE 6275, $62750 \mathrm{U}(2006)$.

[20] Karkare, K. S. et al., "Keck Array and BICEP3: spectral characterization of 5000+ detectors," Proc. SPIE 9153 (2014).

[21] Ahmed, Z. et al., "BICEP3: a next-generation refractor for inflationary CMB polarization," Proc. SPIE 9153 (2014). 